\title{
150. PRESENCIA Y ESTADO DE CONSERVACIÓN DE JASIONE CORYMBOSA POIR. EX SCHULT. (CAMPANULACEAE) EN LA PENÍNSULA IBÉRICA
}

\author{
Miguel SERRANO ${ }^{*}$, Jaime PEREÑA ORTIZ ${ }^{3}$ y Manuel BECERRA PARRA ${ }^{2}$
}

\author{
Recibido el 3 de abril de 2009, aceptado para su publicación el 15 de junio de 2009
} Publicado "on line" en junio de 2009

Presence and conservation status of Jasione corymbosa Poir. ex Schult. (Campanulaceae) in the Iberian Peninsula

Palabras clave. UICN, especie extinta, población remanente, Mediterráneo, flora amenazada.

Key words. IUCN, extinct species, extant population, Mediterranean, threatened flora.

Jasione corymbosa Poir. ex Schult., entendida en sentido amplio, es una especie anual polimorfa endémica del área béticomauritana, cuyo hábitat preferente se situaría sobre dunas y arenales costeros del sur de la península ibérica y del Magreb, desde el occidente de Argelia, hasta la fachada atlántica de Marruecos (Quezel, 1953; Sales \& Hedge, 2001; Peris et al., 2002).

Los registros que atestiguan su presencia en el sur de España son bastante escasos. Existen referencias bibliográficas (Fernández López et al., 1989) y pliegos de herbario, citados en Bokhari \& Sales (2001) y en Sales \& Hedge (2001), que la localizan en la provincia de Málaga, entre Estepona y Fuengirola y en Torrox. De Granada, conocemos un pliego, depositado en el herbario MA, colectado en Calahonda en el año 1984. Esta sería la última cita encontrada hasta el momento, ya que las malagueñas más recientes proceden de inicios del siglo XX. Las referencias a Portugal (Pereira Coutinho, 1939; Parnell, 1987) proceden de confusiones con Jasione montana L. o con Jasione blepharodon Boiss. \& Reut., especie esta última con la que está muy relacionada.

A pesar de que las escasas citas ibéricas hacían presumible un estado de conservación poco alentador para este taxón, no ha sido hasta muy fecha reciente que ha recibido atención en este sentido. Su primera mención en una lista roja aparece en Cabezudo \& Talavera (2005), donde es clasificada en la categoría de amenaza de la UICN (2001) de Datos Insuficientes (DD). Nos consta que los autores de dicho trabajo, antes y después de su publicación, buscaron la especie en su areal histórico, sin resultados. Entendemos que esta es la razón que ha debido motivar su reciente catalogación como especie extinta a nivel regional ibérico (EX, RE) (Moreno, 2008).

Uno de los autores de este trabajo, M. Serrano, también había realizado búsquedas de la especie entre los años 2003 y 2005, siempre infructuosas. Sin embargo, la sospecha de que pudieran quedar remanentes poblacionales en algún punto, principalmente en Málaga dada la mayor presencia de hábitat potencial, llevó a contactar con el personal técnico de flora de la Consejería de Medio Ambiente de la Junta de Andalucía, que puso en marcha un protocolo de localización. Tras un proceso de búsqueda concienzudo, J. corymbosa pudo ser localizada por los otros dos firmantes de este artículo, M. Becerra y J. Pereña, en una sola localidad, perteneciente al municipio de Manilva, en la costa suroccidental malagueña(Pereña Ortiz et al., 2008; Pereña Ortiz et al., 2009) 
Numerosos individuos, en floración e inicios de la fructificación, se acantonaban en la parte mejor conservada de las dunas de Manilva. Los datos de la recolección, depositada en los herbarios de la Universidad de Málaga (MGC) y Universidade de Santiago de Compostela (SANT), son:

MÁLAGA. Manilva. Arenales costeros, 2 m.s.n.m. 13.06.2008, M. Becerra \& J. Pereña. MGC 67726 y SANT 59905; idem: 25.05.2009, B. Cabezudo, A. V. Pérez Latorre \& F. Casimiro-Soriguer. MGC 70.000 (fig.1) y MA s/n.

Esta localidad se encuentra en la provincia corológica Tingitano-Onubo-Algarbiense, Sector Aljíbico (Galán de Mera et al, 2003), y amplía hacia el suroeste el areal peninsular conocido históricamente de esta especie, acercándolo al límite con la provincia de Cádiz.

La complejidad del género Jasione y la escasez de caracteres de discriminación interespecíficos, sumada a la marcada variabilidad intraespecífica de $J$. corymbosa en las poblaciones de Marruecos, suscitó ciertas dudas en el momento de la localización sobre la correcta adscripción de las plantas de Manilva a esta especie (B. Cabezudo, com. pers.)

El material fue estudiado desde las perspectivas molecular y morfológica en el Departamento de Botánica de la Universidade de Santiago de Compostela. Dado que ya disponíamos de secuencias de fragmentos del ADN nuclear ribosómico y del ADN cloroplástico de individuos de $J$. corymbosa procedentes de diversas localidades norteafricanas, se procedió a realizar una extracción de ADN de tejidos foliares de tres plantas de Manilva y a la amplificación por la técnica de PCR de dichas regiones. Los fragmentos empleados del ADN nuclear ribosómico fueron los ITS (Internal Transcribed Spacer) 1 y 2, así como el gen 5.8S. Para el ADN cloroplástico escogimos el espaciador intergénico $p s b A$-trnH y el gen de la subunidad

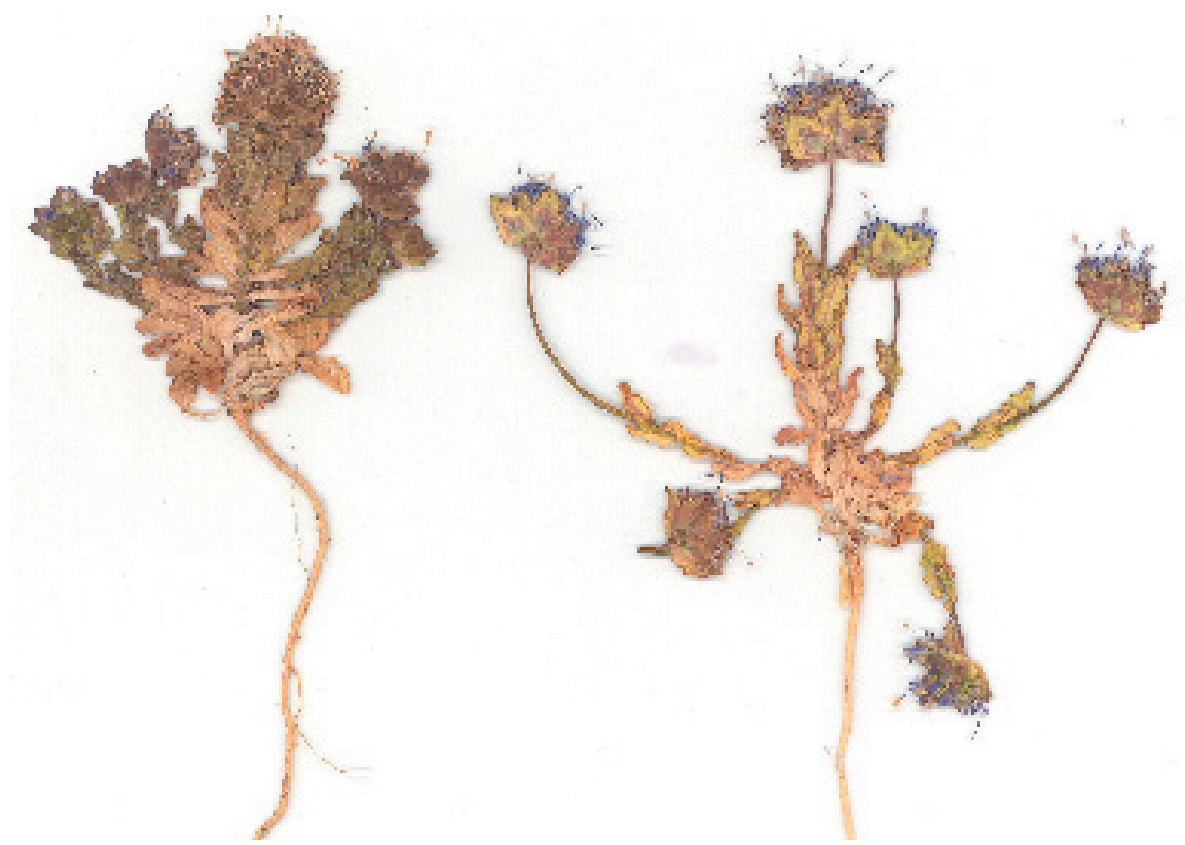

Figura 1. Jasione corymbosa Poir. ex Schult. Málaga: Manilva (MGC 70.000). 
F de la proteína NADH deshidrogenasa $(n d h F)$. Todos ellos ya habían resultado informativos para discriminar a las poblaciones $J$. corymbosa del norte de África de otras especies próximas (Serrano, en prep.).

Los resultados de diversos análisis filogenéticos (Máxima Parsimonia, Bayesianos), en el marco de un estudio amplio que abarca a todo el género (Serrano, en prep.), revelan que las secuencias de ADN amplificadas procedentes de Manilva forman parte de un clado en el que se integran todas las muestras del grupo de $J$. corymbosa, procedentes del norte de África. Estas relaciones inferidas presentan un fuerte apoyo estadístico y sugieren que $J$. corymbosa sería el grupo hermano de otra especie de la mitad sur peninsular, J. blepharodon Boiss. \& Reut. Esta afinidad filogenética entre ambos taxones ya había sido apuntada por PérezEspona et. al. (2005).

Por su morfología, los ejemplares de $J$. corymbosa recolectados en Andalucía no pueden ser claramente diferenciadas de algunas poblaciones norteafricanas, principalmente de las de Rif occidental. Sin embargo, estudios más detallados son necesarios para evaluar en el futuro una posible entidad taxonómica diferenciada, dado el amplio rango de variación que presenta la especie en su conjunto.

\section{Consideraciones sobre su estado de conser- vación.}

Lo restringido de su distribución ibérica, la aparente desaparición de las restantes localidades conocidas, así como la fuerte regresión que padecen las comunidades vegetales de duna en las que se inscribe $J$. corymbosa, amenazadas por la acusada turistificación del litoral mediterráneo andaluz, aconsejan proponer para la península ibérica la categoría de amenaza de En Peligro Crítico (CR), según la clasificación de la UICN (2001). Se modifica, pues, la propuesta de la Lista Roja 2008 de la Flora Vascular Española (Moreno, 2008) en el siguiente sentido: CR B1ab(i,ii,iii)+2 ab(i,ii,iii).
Las primeras medidas de conservación han comenzado a tomarse, consistiendo en el depósito de semillas en los bancos de germoplasma del Jardín Botánico de Córdoba (BGVA), de la Universidad Politécnica de Madrid y de la Universidade de Santiago de Compostela. El hallazgo de esta planta en Manilva permite ser moderadamente optimista ante la posibilidad de que la especie pueda seguir existiendo en algunas otras localidades, lo que hace recomendable seguir buscándola en el litoral andaluz.

AGRADECIMIENTOS. Queremos agradecer a los conservadores de los herbarios B, BRNU, HUAL, K, MA, MAF, RNG y SANT por su ayuda al facilitarnos el préstamo de los especímenes cotejados. Agradecemos también la colaboración e interés en la localización de la especie demostrados por la Consejería de Medio Ambiente de la Junta de Andalucía, particularmente a Guillermo Ceballos y Carmen Rodríguez, así como al agente de Medio Ambiente José Francisco Pérez por su ayuda en el trabajo de campo. Por último, agradecemos por sus valiosos comentarios sobre el manuscrito original a dos revisores anónimos y al editor de esta revista (B. Cabezudo).

\section{BIBLIOGRAFÍA}

BOKHARI, M. \& F. SALES -2001- Jasione anatomy in the Iberian peninsula and its taxonomic significance. Edinburgh Journal of Botany 58: 405-422.

CABEZUDO, B. \& S. TALAVERA(Coordinadores) -2005- Lista roja de la flora vascular de Andalucía. Junta de Andalucía. Sevilla.

FERNÁNDEZ LÓPEZ, C., M. LÓPEZ PULIDO, C. AMEZCUA OGAYAR \& D. CASADO PONCE -1989- Catálogo bibliográfico de las plantas vasculares de Andalucía. Blancoana 7: 3-68.

GALÁN de MERA, A. V. PÉREZ LATORRE y J. V. ORELLANA- 2003- Relaciones fitogeográficas entre el suroccidente de la Península Ibérica y el Noroeste de África: una propuesta de sectorización. Lagascalia 23: 27-51

PARNELL, J. -1987- Variation in Jasione montana L. (Campanulaceae) and related species in Europe and North Africa. Watsonia. 16, 249-267. 
PEREIRA COUTINHO, A.X. -1974- Flora de Portugal: plantas vasculares.- $2^{\mathrm{a}}$ ed.- Bertrand. Lisboa.

PEREÑA ORTIZ, J., M. BECERRA PARRA, A. RIVAS RANGEL y M. SERRANO -2009- Sobre la presencia de Jasione corymbosa Poir. ex Schult. (Campanulaceae) en la península Ibérica. Conservación Vegetal 13:14-15.

PEREÑA ORTIZ, J., M. BECERRA PARRA, A. RIVAS RANGEL y M. SERRANO -2008Jasione corymbosa Poir. ex Schult. Localización de una especie extinta. Medio Ambiente (Junta de Andalucía) 62: 34-35.

PÉREZ-ESPONA, S., F. SALES, I. HEDGE \& M. MÖLLER -2005- Phylogeny and species relationships in Jasione (Campanulaceae) with emphasis on the 'montana-complex'. Edinburgh Journal of Botany 62 (1\&2):29-51.

PERIS, J., A. ROMO \& G. STÜBING -2002Jasione. In B. Valdés, M. Rejdali, A. Achhal, S.L. Jury \& J.M. Montserrat (eds.). Check-list of vascular plants of northern Morocco with identification keys II: 597-598. Consejo Superior de Investigaciones Cientificas.Madrid.

QUÉZEL, P -1953- Les Campanulacées d'Afrique du Nord. Feddes Repertorium Specierum Novarum Regni Vegetabilis 56:1-65.

SALES, F \& I.C. HEDGE -2001-Jasione L. in: Paiva, J., F. Sales, I.C. Hedge, C. Aedo, J.J.Aldasoro, S. Castroviejo, A. Herrero \& M. Velayos, eds. Flora Iberica v. XVI MyoporaceaeCampanulaceae. Real Jardín Botánico de Madrid, CSIC. Madrid.

UICN. 2001. Categorías y criterios de la lista roja de la UICN: versión 3.1. Comisión de Supervivencia de Especies de la UICN. Gland, Suiza y Cambridge, Reino Unido.

Dirección de los autores. ${ }^{1}$ Departamento de Botánica. Faculdade de Farmácia. Universidade de Santiago de Compostela. 15782. Compostela. ${ }^{2}$ Jardín Botánico El Castillejo. Avda. El Castillejo, s/n. 11670 El Bosque (Cádiz). ${ }^{3}$ Delegación Provincial de Medio Ambiente. Junta de Andalucía. Málaga.

* Autor para correspondencia: miguel.serrano@usc.es

151. SOBRE EL GÉNERO EUPHRASIA L. (SCROPHULARIACEAE) EN EL SUR DE LA PENÍSULA IBERICA

Francisco J. PÉREZ-GARCÍA* y Juan F. MOTA

Recibido el 1 de junio de 2009, aceptado para su publicación el 15 de junio de 2009 Publicado "on line" en octubre de 2009

On the genus Euphrasia (Scrophulariaceae) L. in Southern Spain

Palabras clave. Biodiversidad, corología, Montañas Béticas, Sureste ibérico.

Key words. Biodiversity, Chorology, Baetic Ranges, South-eastern Iberian Peninsula.

El género Euphrasia L. está representado en la Península Ibérica por nueve especies
(Vitek, 2008), de las cuales solo dos están presentes en Andalucía y por ende en la 